\title{
Finite difference scheme for simulating a generalized two-dimensional multi-term time fractional non-Newtonian fluid model
}

\author{
Yanqin Liu', ${ }^{1,2}$, Xiuling Yin ${ }^{1}$, Libo Feng ${ }^{3}$ and Hongguang Sun ${ }^{2}$
}

\section{"Correspondence:}

yqliumath@163.com

'School of Mathematical Sciences, Dezhou University, Dezhou, China

${ }^{2}$ Institute of Soft Matter Mechanics, Department of Engineering

Mechanics, Hohai University, Jiangsu, China

Full list of author information is available at the end of the article

\section{空 Springer}

\begin{abstract}
A finite difference scheme, based upon the Crank-Nicolson scheme, is applied to the numerical approximation of a two-dimensional time fractional non-Newtonian fluid model. This model not only possesses a multi-term time derivative, but also contains a special time fractional operator on the spatial derivative. And a very important lemma is proposed and also proved, which plays a vital role in the proof of the unconditional stability. The stability and convergence of the finite difference scheme are discussed and theoretically proved by the energy method. Numerical experiments are given to validate the accuracy and efficiency of the scheme, and the results indicate that this Crank-Nicolson difference scheme is very effective for simulating the generalized non-Newtonian fluid diffusion model.
\end{abstract}

Keywords: Finite difference method; Crank-Nicolson difference scheme; Energy method; Caputo fractional derivative; Multi-term time derivative; Generalized non-Newtonian fluid

\section{Introduction}

Fractional partial differential equations have been applied to many anomalous phenomena and complex systems in natural science and engineering technology fields [1-3]. They have some advantages in describing real processes or phenomena with memory $[4,5]$ over the integer-order ones. Many existing models have used single-term time-fractional derivatives to describe anomalous diffusion phenomena [6, 7], optimum control [8, 9], ecological system [10,11]. In recent years, the extension to multi-term time fractional diffusion equations has been considered. Liu et al. [12] derived the analytical solutions for the multi-time time-fractional diffusion-wave equations with nonhomogeneous boundary conditions by using the method of separating variables. Daftardar-Gejji et al. [13] obtained the solutions of multi-term time fractional diffusion wave equations under different homogeneous or non-homogeneous boundary conditions. Some numerical treatment of multi-term fractional diffusion equations is also an active area of current research. The Galerkin finite element method was discussed in [14]. Liu et al. [15] found numerical solutions for the multi-term fractional diffusion-wave diffusion whose fractional orders belong to the intervals $[0,1),[1,2),[0,2),[0,3),[2,3)$, and $[2,4)$, separately. In [16], they handled the multi-term fractional wave equations with a meshless collocation method

(c) The Author(s) 2018. This article is distributed under the terms of the Creative Commons Attribution 4.0 International License (http://creativecommons.org/licenses/by/4.0/), which permits unrestricted use, distribution, and reproduction in any medium, provided you give appropriate credit to the original author(s) and the source, provide a link to the Creative Commons license, and indicate if changes were made. 
and applied the moving least squares reproducing kernel particle approximation to construct the shape functions for spatial approximation of the equations in two dimensions. An implicit compact difference scheme based on the $L_{2}$ approximation was constructed to simulate the solution of the two-dimensional multi-term fractional equations in [17].

Fractional equations also have many other applications, such as continuous time random walks in external fields [18], fractal mobile/immobile solute transport [19], attenuation coefficient for power-law biological tissue [20]. In the last few decades, non-Newtonian fluids have been widely applied in engineering and industry. The constitutive equation of non-Newtonian fluids is much more complex than its Newtonian counterparts, and the constitutive equations involving fractional derivative have been proved to be a valuable tool to handle viscoelastic properties [21], and the obtained results show that they are in good agreement with the experimental data [22].

The generalized Oldroyd-B fluid is a particular subclass of non-Newtonian fluids. Some recent papers about the generalized Oldroyd-B fluids can be found in references [23, 24]. The fundamental electromagnetic relations have been summarized by Sutton [25]. One important part is the incompressible Oldroyd-B fluid which is bounded by two infinite rigid plates, when a magnetic field is imposed on the above flow under the assumption of low magnetic Reynolds number. Khan et al. [26] considered the generalized Oldroyd-B fluid in a porous medium with the influence of Hall current. For the MHD flow, Zheng et al. [27] discussed the flow between two plates with slip boundary conditions and obtained the exact solution using some transform techniques. There are some literature works [27, 28 ] that give the exact solution of the generalized Oldroyd-B fluid, and they are typically given in generalized $\mathrm{G}$ or $\mathrm{H}$-function. Fetecau et al. [29] considered the two-dimensional fluid model and also obtained the exact solution.

But these multi-term fractional fluid models are difficult to get their exact solution, therefore, many researchers look for other solutions [30,31]; numerical method is a promising tool to solve these equations. And up to now, numerical methods to solve fractional equations mainly are finite difference methods [32-34], finite element methods [35, 36], finite volume methods [37, 38], and spectral methods [39-41]. For these multiterm fractional fluid models, Bazhlekova et al. [42] proposed a finite difference method to solve the viscoelastic flow with generalized fractional Oldroyd-B model, and they utilized the Grünwald-Letnikov formula to approximate the Riemann-Liouville time fractional derivative; the results were low accuracy and lacked theoretical analysis. Recently, Feng et al. [43] gave the numerical solution of these problems, but it was confined to a one-dimensional case. We use finite difference method to solve the generalized twodimensional multi-term time fractional Oldroyd-B fluid equation. We get that the temporal convergence order is $\min \{3-\gamma, 2-\alpha, 2-\beta\}$, this result is better than [43] where the convergence rate in time is only first order, and we also propose and prove a vital lemma. It will help us to prove the unconditional stability of the Crank-Nicolson finite difference scheme, but in literature [44], they only proved that this Crank-Nicolson difference scheme is conditionally stable.

Inspired by the above works and motivated by potential applications, we will consider the following generalized two-dimensional multi-term time fractional non-Newtonian fluid diffusion equation:

$$
c_{10}^{C} D_{t}^{\gamma} u+c_{2} \frac{\partial u}{\partial t}+c_{30}^{C} D_{t}^{\alpha} u+c_{4} u=c_{5} \Delta u+c_{60}^{C} D_{t}^{\beta} \Delta u+f(\mathbf{x}, t)
$$


where $u=u(\mathbf{x}, t), \mathbf{x}=(x, y) \in \Omega=\left(0, L_{x}\right) \times\left(0, L_{y}\right) \subset \mathfrak{R}^{2}, 0<t \leq T$, and the following initial conditions:

$$
u(\mathbf{x}, 0)=\varphi(\mathbf{x}), \quad u_{t}(\mathbf{x}, 0)=\phi(\mathbf{x}), \quad \mathbf{x} \in \Omega
$$

and Dirichlet boundary condition

$$
u(\mathbf{x}, t)=0, \quad \mathbf{x} \in \partial \Omega \subset \mathfrak{R}^{2}, 0<t \leq T,
$$

where $c_{i}>0, i=1, \ldots, 6$, and $1<\gamma<2,0<\alpha, \beta<1$, here $\partial \Omega$ is the boundary of $\Omega$. $\triangle$ is the Laplace operator $\Delta u=\frac{\partial^{2} u}{\partial x^{2}}+\frac{\partial^{2} u}{\partial y^{2}}, \varphi(\mathbf{x})$ and $\phi(\mathbf{x})$ are sufficiently smooth functions, $f(\mathbf{x}, t)$ is a known source term function. ${ }_{0}^{C} D_{t}^{\theta} u$ is the Caputo time fractional derivative of order $\theta(\theta>0, n-1 \leq \theta<n)$ with respect to $t[45,46]$

$$
{ }_{0}^{C} D_{t}^{\theta} u(\mathbf{x}, t)= \begin{cases}\frac{1}{\Gamma(n-\theta)} \int_{0}^{t}(t-s)^{n-1-\theta} \frac{\partial^{n} u(\mathbf{x}, s)}{\partial s^{n}} d s, & \theta \notin \mathbf{z}^{+}, \\ \frac{\partial^{\theta} u(\mathbf{x}, t)}{\partial t^{\theta}}, & \theta \in \mathbf{z}^{+},\end{cases}
$$

where $\Gamma(\cdot)$ is the gamma function.

The outline of this paper is organized as follows. First, preliminary knowledge is given, and the numerical discretization of the time fractional derivative is proposed. Then, we develop the finite difference method for the generalized non-Newtonian fluid model and derive the implicit scheme. After that, we proceed with the proof of the stability and convergence of the scheme by energy method and discuss the solvability of the numerical scheme. Finally, we present a numerical example to demonstrate the effectiveness of our method and draw some conclusions.

\section{Preliminary}

In the $x$-direction $\left[0, L_{p}\right]$, we take the mesh points $x_{p}=p h_{1}, p=0,1, \ldots, M_{1}$, in the $y$ direction $\left[0, L_{q}\right]$, we take the mesh points $y_{q}=q h_{1}, q=0,1, \ldots, M_{1}$, and $t_{n}=n \tau, n=$ $0,1, \ldots, N$, where $h_{x}=L_{p} / M_{1}, h_{y}=L_{q} / M_{1}, \tau=T / N$ are the uniform spatial step size and temporal step size, respectively. Denote $\Omega_{\tau} \equiv\left\{t_{n} \mid 0 \leq n \leq N\right\}, \Omega_{h} \equiv\left\{\left(x_{p}, y_{q}\right) \mid 0 \leq p \leq\right.$ $\left.M_{1}, 0 \leq q \leq M_{2}\right\}$. Suppose $u_{p q}^{n}=u\left(x_{p}, y_{q}, t_{n}\right), u_{p q}^{n}$ is a grid function on $\Omega_{h} \times \Omega_{\tau}$. We introduce the following notations:

$$
\begin{aligned}
& \nabla_{t} u_{p q}^{n}=\frac{u_{p q}^{n}-u_{p q}^{n-1}}{\tau}, \quad u_{p q}^{n-\frac{1}{2}}=\frac{u_{p q}^{n}+u_{p q}^{n-1}}{2}, \quad \nabla_{x} u_{p q}^{n}=\frac{u_{p q}^{n}-u_{p-1 q}^{n}}{h}, \\
& \delta_{x}^{2} u_{p q}^{n}=\frac{u_{p-1 q}^{n}-2 u_{p q}^{n}+u_{p+1 q}^{n}}{h_{x}^{2}}, \quad \delta_{y}^{2} u_{p q}^{n}=\frac{u_{p q-1}^{n}-2 u_{p q}^{n}+u_{p q+1}^{n}}{h_{y}^{2}} .
\end{aligned}
$$

We define $\mathcal{V}_{h}=\left\{v \mid v\right.$ is a grid function on $\Omega_{h}$ and $\left.v_{0 q}=v_{M_{x} q}=v_{p 0}=v_{p M_{y}}=0\right\}$. For any $u, v \in \mathcal{V}_{h}$, we also define the following discrete inner products and induced norms:

$$
\begin{aligned}
& (u, v)=h_{x} h_{y} \sum_{p=1}^{M_{x}-1} \sum_{p=1}^{M_{y}-1} u_{p q} v_{p q}, \\
& \left\langle\left(\nabla_{x}+\nabla_{y}\right) u,\left(\nabla_{x}+\nabla_{y}\right) v\right\rangle=h_{x} h_{y} \sum_{p=1}^{M_{x}} \sum_{p=1}^{M_{y}}\left(\nabla_{x}+\nabla_{y}\right) u_{p q} \cdot\left(\nabla_{x}+\nabla_{y}\right) v_{p q},
\end{aligned}
$$




$$
\|v\|_{0}=\sqrt{(v, v)}, \quad\|v\|_{\infty}=\max _{\substack{1 \leq p \leq M_{x} \\ 1 \leq q \leq M_{y}}}|v|, \quad|v|_{1}=\sqrt{\left\langle\left(\nabla_{x}+\nabla_{y}\right) v,\left(\nabla_{x}+\nabla_{y}\right) v\right|} .
$$

The $\|\cdot\|_{1}$ norm of the grid function $v \in \mathcal{V}_{h}$ is given as

$$
\|v\|_{1}=\sqrt{c_{4}\|v\|_{0}^{2}+c_{5}\|v\|_{1}^{2}} .
$$

For the two-dimensional case, we can get the following properties:

$$
\begin{aligned}
& \begin{aligned}
\left(\left(\delta_{x}^{2}+\delta_{y}^{2}\right) v^{k}, v^{n}\right)= & -\left\langle\left(\nabla_{x}+\nabla_{y}\right) v^{k},\left(\nabla_{x}+\nabla_{y}\right) v^{n}\right\rangle, \\
\left(\left(\delta_{x}^{2}+\delta_{y}^{2}\right) v^{k}, \nabla_{t} v^{n}\right) & =-\frac{1}{\tau}\left\langle\left(\nabla_{x}+\nabla_{y}\right) v^{k},\left(\nabla_{x}+\nabla_{y}\right) v^{n}-\left(\nabla_{x}+\nabla_{y}\right) v^{n-1}\right\rangle \\
& =-\left\langle\left(\nabla_{x}+\nabla_{y}\right) v^{k}, \nabla_{t}\left(\nabla_{x}+\nabla_{y}\right) v^{n}\right\rangle,
\end{aligned} \\
& \left(\nabla_{t}\left(\left(\delta_{x}^{2}+\delta_{y}^{2}\right) v^{k}\right), \nabla_{t} v^{n}\right)=-\left\langle\nabla_{t}\left(\left(\nabla_{x}+\nabla_{y}\right) v^{k}\right), \nabla_{t}\left(\left(\nabla_{x}+\nabla_{y}\right) v^{n}\right)\right\rangle .
\end{aligned}
$$

Proof We only give the proof of formula (4), similar to the one-dimensional case, it is easy to get

$$
\begin{aligned}
& \left(\nabla_{t}\left(\left(\delta_{x}^{2}+\delta_{y}^{2}\right) v^{k}\right), \nabla_{t} v^{n}\right) \\
& =\frac{1}{\tau}\left\langle\left(\nabla_{x}+\nabla_{y}\right) v^{k}-\left(\nabla_{x}+\nabla_{y}\right) v^{k-1}, \nabla_{t} v^{n}\right\rangle \\
& =-\frac{1}{\tau}\left(\left\langle\left(\nabla_{x}+\nabla_{y}\right) v^{k}, \nabla_{t}\left(\nabla_{x}+\nabla_{y}\right) v^{n}\right\rangle-\left\langle\left(\nabla_{x}+\nabla_{y}\right) v^{k-1}, \nabla_{t}\left(\nabla_{x}+\nabla_{y}\right) v^{n}\right\rangle\right) \\
& =-\left\langle\nabla_{t}\left(\left(\nabla_{x}+\nabla_{y}\right) v^{k}\right), \nabla_{t}\left(\left(\nabla_{x}+\nabla_{y}\right) v^{n}\right)\right\rangle .
\end{aligned}
$$

Next we will give the Crank-Nicolson scheme of fractional derivative.

\subsection{Crank-Nicolson discretization of $D_{t}^{\beta} u(0<\beta<1)$}

From the Crank-Nicolson scheme in [44, 47], we give the discretization scheme at grid points $\left(x_{p}, y_{q}, t_{n-\frac{1}{2}}\right)$ directly

$$
\begin{aligned}
D_{t}^{\beta} u\left(x_{p}, y_{q}, t_{n-\frac{1}{2}}\right) & =\frac{\tau^{-\beta}}{\Gamma(2-\beta)}\left[d_{0}^{(\beta)} u_{p q}^{n}-\sum_{k=1}^{n-1}\left(d_{n-k-1}^{(\beta)}-d_{n-k}^{(\beta)}\right) u_{p q}^{k}-d_{n-1}^{(\beta)} u_{p q}^{0}\right]+R_{1} \\
& =\frac{\tau^{1-\beta}}{\Gamma(2-\beta)} \sum_{k=1}^{n} d_{n-k}^{(\beta)} \nabla_{t} u_{p q}^{k}+R_{1},
\end{aligned}
$$

where $R_{1} \leq C\left(\tau^{2-\beta}\right)$, and

$$
d_{k}^{(\beta)}= \begin{cases}\left(k+\frac{1}{2}\right)^{1-\beta}-\left(k-\frac{1}{2}\right)^{1-\beta}, & k=1,2,3, \ldots, \\ 2^{\beta-1}, & k=0 .\end{cases}
$$

Lemma 1 The coefficients $d_{k}^{(\beta)}$ satisfy the following properties:

(1) $d_{k}^{(\beta)}>0, \lim _{k \rightarrow \infty} d_{k}^{(\beta)}=0$,

(2) $d_{k}^{(\beta)}>d_{k+1}^{(\beta)}, k \geq 1$,

(3) $d_{k+1}^{(\beta)}-2 d_{k}^{(\beta)}+d_{k-1}^{(\beta)}>0, k \geq 2$. 
Remark 1 When $0<\beta<0.4811, d_{2}^{(\beta)}-2 d_{1}^{(\beta)}+d_{0}^{(\beta)}<0$. Regarding these coefficients' property, literature [44] proved that this Crank-Nicolson difference scheme is only conditionally stable. Next, we will give the following important lemma, which is a valuable tool to prove the unconditional stability of this Crank-Nicolson difference scheme.

Lemma 2 For $0<\beta<1, d_{k}^{(\beta)}$ are defined as (5), (6), and for any positive integer $N$ and real vector $\mathbf{Q}=\left(v^{1}, v^{2}, \ldots, v^{N-1}, v^{N}\right) \in R^{N+1}$, we have

$$
\sum_{n=1}^{N} \sum_{k=1}^{n} d_{n-k}^{(\beta)} v^{k} v^{n} \geq 0
$$

Proof From Remark 1, when $0<\beta<0.4811$, the coefficient $d_{2}^{(\beta)}-2 d_{1}^{(\beta)}+d_{0}^{(\beta)}<0$. Next, we will give the proof that it does not affect the results. Then, similar to Feng's proof [48], we will first prove the following results. We can rewrite (7) in the following form:

$$
\sum_{n=1}^{N} \sum_{k=1}^{n} d_{n-k}^{(\beta)} v^{k} v^{n}=\sum_{n=1}^{N} \sum_{k=0}^{n-1} d_{n-k}^{(\beta)} v^{n-k} v^{n}=\mathbf{Q} \mathbf{W} \mathbf{Q}^{T}
$$

where

$$
\mathbf{W}=\left[\begin{array}{cccccc}
d_{0}^{(\beta)} & 0 & 0 & \cdots & 0 & 0 \\
d_{1}^{(\beta)} & d_{0}^{(\beta)} & 0 & \cdots & 0 & 0 \\
d_{2}^{(\beta)} & d_{1}^{(\beta)} & d_{0}^{(\beta)} & \cdots & 0 & 0 \\
\vdots & \vdots & \vdots & \ddots & \vdots & \vdots \\
d_{N-2}^{(\beta)} & d_{N-3}^{(\beta)} & d_{N-4}^{(\beta)} & \cdots & d_{0}^{(\beta)} & 0 \\
d_{N-1}^{(\beta)} & d_{N-2}^{(\beta)} & d_{N-3}^{(\beta)} & \cdots & d_{1}^{(\beta)} & d_{0}^{(\beta)}
\end{array}\right]
$$

To prove (7) is equivalent to proving that Matrix $\mathbf{W}$ is positive definite. So we only need to prove $\mathbf{T}_{\mathbf{N}}=\frac{\mathbf{W}+\mathbf{W}^{T}}{2}$ is positive definite [43]. $\mathbf{T}_{\mathbf{N}}$ is a real symmetric Toeplitz matrix and has the form

$$
\mathbf{T}_{\mathbf{N}}=\frac{1}{2}\left[\begin{array}{cccccc}
2 d_{0}^{(\beta)} & d_{1}^{(\beta)} & d_{2}^{(\beta)} & \cdots & d_{N-2}^{(\beta)} & d_{N-1}^{(\beta)} \\
d_{1}^{(\beta)} & 2 d_{0}^{(\beta)} & d_{1}^{(\beta)} & \cdots & d_{N-3}^{(\beta)} & d_{N-2}^{(\beta)} \\
d_{2}^{(\beta)} & d_{1}^{(\beta)} & 2 d_{0}^{(\beta)} & \cdots & d_{N-4}^{(\beta)} & d_{N-3}^{(\beta)} \\
\vdots & \vdots & \vdots & \ddots & \vdots & \vdots \\
d_{N-2}^{(\beta)} & d_{N-3}^{(\beta)} & d_{N-4}^{(\beta)} & \cdots & 2 d_{0}^{(\beta)} & d_{1}^{(\beta)} \\
d_{N-1}^{(\beta)} & d_{N-2}^{(\beta)} & d_{N-3}^{(\beta)} & \cdots & d_{1}^{(\beta)} & 2 d_{0}^{(\beta)}
\end{array}\right]
$$

Next, we will prove $\operatorname{det}\left(\mathbf{T}_{\mathbf{N}}\right)>0$. It is easy to verify that $\operatorname{det}\left(\mathbf{T}_{\mathbf{1}}\right)=2 d_{0}^{(\beta)}>0$, and $\operatorname{det}\left(\mathbf{T}_{\mathbf{2}}\right)=$ $2\left(d_{0}^{(\beta)}\right)^{2}-\frac{1}{2} d_{1}^{(\beta)}>0$, for a finite integer $N$, we explicitly calculate the value of $\operatorname{det}\left(\mathbf{T}_{\mathbf{N}}\right)>0$. When $N$ is sufficiently large, according to [49], we have

$$
\frac{\operatorname{det}\left(\mathbf{T}_{\mathbf{N}}\right)}{\operatorname{det}\left(\mathbf{T}_{\mathbf{N}+\mathbf{1}}\right)}>0
$$


then we can conclude that $\operatorname{det}\left(\mathbf{T}_{\mathbf{N}+\mathbf{1}}\right)>0$. As matrix $\mathbf{T}_{\mathbf{k}}, \mathbf{k}=\mathbf{1}, \mathbf{2}, \ldots, \mathbf{N}$ are the principal minors of matrix $H_{N+1}$ and $\operatorname{det}\left(\mathbf{T}_{\mathbf{k}}\right)>0, k=1,2, \ldots, N+1$, then the real symmetric Toeplitz matrix $\mathbf{T}_{\mathbf{N}+\mathbf{1}}$ is positive definite, matrix $\mathbf{W}$ is positive definite, so (7) is proved.

In this part, we will give the scheme of the fractional derivative $D_{t}^{\beta} \Delta u, 0<\beta<1$.

Since

$$
\begin{aligned}
\Delta u\left(x_{p}, y_{q}, t_{n-\frac{1}{2}}\right)= & \left(\delta_{x}^{2}+\delta_{y}^{2}\right) u\left(x_{p}, y_{q}, t_{n-\frac{1}{2}}\right) \\
& -\frac{h_{x}^{2}}{12} \frac{\partial^{4} u\left(\xi_{p}, y_{q}, t_{n-\frac{1}{2}}\right)}{\partial x^{4}}-\frac{h_{y}^{2}}{12} \frac{\partial^{4} u\left(x_{p}, \varsigma_{q}, t_{n-\frac{1}{2}}\right)}{\partial y^{4}},
\end{aligned}
$$

where $x_{p-1} \leq \xi_{p} \leq x_{p}, y_{q-1} \leq \varsigma_{q} \leq y_{q}$, then

$$
D_{t}^{\beta} \Delta u\left(x_{p}, y_{q}, t_{n-\frac{1}{2}}\right)=\frac{\tau^{1-\beta}}{\Gamma(2-\beta)} \sum_{k=1}^{n} d_{n-k}^{(\beta)} \nabla_{t}\left(\delta_{x}^{2}+\delta_{y}^{2}\right) u_{p q}^{k}+R_{2}
$$

where $\left|R_{2}\right| \leq C \tau^{2-\beta}+h_{x}^{2}+h_{y}^{2}$.

Next we give the following lemmas [50].

Lemma 3 Discretization of the time fractional derivative $D_{t}^{\gamma} u(x, y, t)(1<\gamma<2)$. From the results in [50], at mesh points $\left(x_{p}, y_{q}, t_{n-\frac{1}{2}}\right)$ we get

$$
\begin{aligned}
D_{t}^{\gamma} u\left(x_{p}, y_{q}, t_{n-\frac{1}{2}}\right) \approx & \frac{1}{2}\left[D_{t}^{\gamma} u\left(x_{p}, y_{q}, t_{n}\right)+D_{t}^{\gamma} u\left(x_{p}, y_{q}, t_{n-1}\right)\right] \\
= & \frac{\tau^{1-\gamma}}{\Gamma(3-\gamma)}\left[a_{0}^{(\gamma)} \nabla_{t} u_{p q}^{n}-\sum_{k=1}^{n-1}\left(a_{n-k-1}^{(\gamma)}-a_{n-k}^{(\gamma)}\right) \nabla_{t} u_{p q}^{k}\right. \\
& \left.-a_{n-1}^{(\gamma)} \frac{\partial u\left(x_{p}, y_{q}, 0\right)}{\partial t}\right]+R_{3},
\end{aligned}
$$

where $R_{3}=O\left(\tau^{3-\gamma}\right)$, the coefficients $a_{k}^{(\gamma)}=(k+1)^{2-\gamma}-k^{2-\gamma}, k=0,1,2, \ldots$, and they satisfy the following properties:

(1) $a_{k}^{(\gamma)}>0, a_{0}^{(\gamma)}=1, a_{k}^{(\gamma)}>a_{k+1}^{(\gamma)}, \lim _{k \rightarrow \infty} a_{k}^{(\gamma)}=0$,

(2) $\sum_{k=0}^{n-1}\left(a_{k}^{(\gamma)}-a_{k+1}^{(\gamma)}\right)+a_{n}^{(\gamma)}=1$,

(3) $(2-\gamma)(k+1)^{1-\gamma} \leq a_{k}^{(\gamma)} \leq(2-\gamma) k^{1-\gamma}$.

Lemma $4([43,50])$ For $1<\gamma<2$, define $a_{k}^{(\gamma)}=(k+1)^{2-\gamma}-k^{2-\gamma}, k=0,1,2, \ldots, n$, and $S=\left\{S_{1}, S_{2}, S_{3}, \ldots\right\}$ and $P$, then it holds that

$$
\begin{aligned}
& \frac{\tau^{1-\gamma}}{\Gamma(3-\gamma)} \sum_{n=1}^{N}\left[a_{0}^{(\gamma)} S_{n}-\sum_{k=1}^{n-1}\left(a_{n-k-1}^{(\gamma)}-a_{n-k}^{(\gamma)}\right) S_{k}-a_{n-1}^{(\gamma)} P\right] S_{n} \\
& \quad \geq \frac{T^{1-\gamma}}{2 \Gamma(2-\gamma)} \sum_{n=1}^{N} S_{n}^{2}-\frac{T^{2-\gamma}}{2 \tau \Gamma(3-\gamma)} P^{2}, \quad N=1,2,3, \ldots
\end{aligned}
$$




\section{The derivation of the difference scheme}

We assume $u(x, y, t) \in C_{x, y, t}^{4,4,3}(\Omega \times(0, T])$ and define the grid function $f_{p q}^{n}=f\left(x_{p}, y_{q}, t_{n}\right), \varphi_{p q}=$ $\varphi\left(x_{p}, y_{q}\right), \phi_{p q}=\phi\left(x_{p}, y_{q}\right),\left(x_{p}, y_{q}\right) \in \Omega_{h}, 0 \leq n \leq N$. Now, we will present the finite difference scheme for the two-dimensional generalized non-Newtonian fluid model (1)

$$
\begin{gathered}
c_{1} D_{t}^{\gamma} u\left(x_{p}, y_{q}, t_{n-\frac{1}{2}}\right)+c_{2} \frac{\partial u\left(x_{p}, y_{q}, t_{n-\frac{1}{2}}\right)}{\partial t}+c_{3} D_{t}^{\alpha} u\left(x_{p}, y_{q}, t_{n-\frac{1}{2}}\right)+c_{4} u\left(x_{p}, y_{q}, t_{n-\frac{1}{2}}\right) \\
=c_{5} \Delta u\left(x_{p}, y_{q}, t_{n-\frac{1}{2}}\right)+c_{6} D_{t}^{\beta} \Delta u\left(x_{p}, y_{q}, t_{n-\frac{1}{2}}\right)+f\left(x_{p}, y_{q}, t_{n-\frac{1}{2}}\right) .
\end{gathered}
$$

From Eqs. (5) and (8), one gets

$$
\begin{aligned}
c_{1} \mu_{1}[ & \left.a_{0}^{(\gamma)} \nabla_{t} u_{p q}^{n}-\sum_{k=1}^{n-1}\left(a_{n-k-1}^{(\gamma)}-a_{n-k}^{(\gamma)}\right) \nabla_{t} u_{p q}^{k}-a_{n-1}^{(\gamma)} \phi_{p q}\right] \\
& +c_{2} \nabla_{t} u_{p q}^{n}+c_{3} \mu_{2} \sum_{k=1}^{n} d_{n-k}^{(\alpha)} \nabla_{t} u_{p q}^{k}+c_{4} u_{p q}^{n-\frac{1}{2}} \\
= & c_{5}\left(\delta_{x}^{2}+\delta_{y}^{2}\right) u_{p q}^{n-\frac{1}{2}}+c_{6} \mu_{3} \sum_{k=1}^{n} d_{n-k}^{(\beta)} \nabla_{t}\left(\delta_{x}^{2}+\delta_{y}^{2}\right) u_{p q}^{k} \\
& +f_{p q}^{n-\frac{1}{2}}+R_{p q}^{n}
\end{aligned}
$$

where $\mu_{1}=\frac{\tau^{1-\gamma}}{\Gamma(3-\gamma)}, \mu_{2}=\frac{\tau^{1-\alpha}}{\Gamma(2-\alpha)}, \mu_{3}=\frac{\tau^{1-\beta}}{\Gamma(2-\beta)}$, and $u\left(x_{p}, y_{q}, t_{n-\frac{1}{2}}\right)=\frac{u\left(x_{p}, y_{q}, t_{n}\right)+u\left(x_{p}, y_{q}, t_{n-1}\right)}{2}+O\left(\tau^{2}\right)$, $\frac{\partial}{\partial t} u\left(x_{p}, y_{q}, t_{n-\frac{1}{2}}\right)=\frac{u\left(x_{p}, y_{q}, t_{n}\right)-u\left(x_{p}, y_{q}, t_{n-1}\right)}{\tau}+O\left(\tau^{2}\right)$, so $\left|R_{p q}^{n}\right| \leq C\left(\tau^{\min (3-\gamma, 2-\alpha, 2-\beta)}+h_{1}^{2}+h_{2}^{2}\right)$, in which $C$ is independent of $\tau, h_{1}$, and $h_{2}$. Omitting the error term, we use $U_{p q}^{n}$ as the numerical solution, then we obtain the implicit finite difference scheme for generalized nonNewtonian fluid Eq. (1)

$$
\begin{aligned}
& c_{l} \mu_{l}\left[a_{0}^{(\gamma)} \nabla_{t} U_{p q}^{n}-\sum_{k=1}^{n-1}\left(a_{n-k-1}^{(\gamma)}-a_{n-k}^{(\gamma)}\right) \nabla_{t} U_{p q}^{k}-a_{n-1}^{(\gamma)} \phi_{p q}\right] \\
& \quad+c_{2} \nabla_{t} U_{p q}^{n}+c_{3} \mu_{2} \sum_{k=1}^{n} d_{n-k}^{(\alpha)} \nabla_{t} U_{p q}^{k}+c_{4} U_{p q}^{n-\frac{1}{2}} \\
& =c_{5}\left(\delta_{x}^{2}+\delta_{y}^{2}\right) U_{p q}^{n-\frac{1}{2}}+c_{6} \mu_{3} \sum_{k=1}^{n} c_{n-k}^{(\beta)} \nabla_{t}\left(\delta_{x}^{2}+\delta_{y}^{2}\right) U_{p q}^{k}+f_{p q}^{n-\frac{1}{2}},
\end{aligned}
$$

with the initial and boundary conditions

$$
\begin{aligned}
& U_{p q}^{0}=\varphi_{p q}, \quad\left(x_{p}, y_{q}\right) \in \Omega_{h}, \\
& U_{p q}^{n}=0, \quad\left(x_{p}, y_{q}\right) \in \mathcal{V}_{h}, 1 \leq n \leq N .
\end{aligned}
$$

\section{Analysis of the numerical scheme}

\subsection{Solvability of the scheme}

Theorem 1 The implicit finite difference scheme (11) is uniquely solvable. 
Proof At each time level, the coefficient matrix $A$ is

$$
A=\left[\begin{array}{cccccc}
B & C & 0 & \cdots & 0 & 0 \\
C & B & C & \cdots & 0 & 0 \\
0 & C & B & \cdots & 0 & 0 \\
\vdots & \vdots & \vdots & \ddots & \vdots & \vdots \\
0 & 0 & 0 & \cdots & B & C \\
0 & 0 & 0 & \cdots & C & B
\end{array}\right]
$$

where $B$ and $C$ are block matrixes,

$$
B=\left[\begin{array}{cccccc}
r_{1}+2 r_{2}+2 r_{3} & -r_{3} & 0 & \cdots & 0 & 0 \\
-r_{3} & r_{1}+2 r_{2}+2 r_{3} & -r_{3} & \cdots & 0 & 0 \\
0 & -r_{3} & r_{1}+2 r_{2}+2 r_{3} & \cdots & 0 & 0 \\
\vdots & \vdots & \vdots & \ddots & \vdots & \vdots \\
0 & 0 & 0 & \cdots & r_{1}+2 r_{2}+2 r_{3} & -r_{3} \\
0 & 0 & 0 & \cdots & -r_{3} & r_{1}+2 r_{2}+2 r_{3}
\end{array}\right]
$$

and

$$
C=\left[\begin{array}{cccccc}
-r_{2} & 0 & 0 & \cdots & 0 & 0 \\
0 & -r_{2} & 0 & \cdots & 0 & 0 \\
0 & 0 & -r_{2} & \cdots & 0 & 0 \\
\vdots & \vdots & \vdots & \ddots & \vdots & \vdots \\
0 & 0 & 0 & \cdots & -r_{2} & 0 \\
0 & 0 & 0 & \cdots & 0 & -r_{2}
\end{array}\right] \text {, }
$$

where $r_{1}=\frac{c_{1} k_{1}+c_{2}+c_{3} k_{2} d_{0}^{(\alpha)}}{\tau}+\frac{c_{4}}{2}>0, r_{2}=\frac{\frac{c_{5}}{2}+c_{6} k_{3} d_{0}^{(\beta)}}{h_{1}^{2}}>0, r_{3}=\frac{\frac{c_{5}}{2}+c_{6} k_{3} d_{0}^{(\beta)}}{h_{2}^{2}}>0, r_{4}=\frac{c_{1} k_{1}+c_{2}}{\tau}-\frac{b_{2}}{2}$, and $r_{5}=\frac{c_{5}}{2}$. Then $A$ is a strictly diagonally dominant matrix. Therefore $A$ is nonsingular, which means that the numerical scheme (11) is uniquely solvable.

\subsection{Stability}

Theorem 2 The implicit finite difference scheme (11) is unconditionally stable.

Proof Multiplying Eq. (11) by $h_{1} h_{2} \tau \nabla_{t} U_{p q}^{n}$ and summing $p$ from 1 to $M_{1}-1, q$ from 1 to $M_{2}-1$ and summing $n$ from 1 to $N$, we obtain

$$
\begin{aligned}
& c_{1} k_{1} \tau \sum_{n=1}^{N} \sum_{p=1}^{M_{1}-1} \sum_{q=1}^{M_{2}-1} h_{1} h_{2}\left[a_{0}^{(\gamma)} \nabla_{t} U_{p q}^{n}-\sum_{k=1}^{n-1}\left(a_{n-k-1}^{(\gamma)}-a_{n-k}^{(\gamma)}\right) \nabla_{t} U_{p q}^{k}-a_{n-1}^{(\gamma)} \phi_{p q}\right] \nabla_{t} U_{p q}^{n} \\
& +c_{2} \tau \sum_{n=1}^{N} \sum_{p=1}^{M_{1}-1} \sum_{q=1}^{M_{2}-1} h_{1} h_{2}\left(\nabla_{t} U_{p q}^{n}\right)^{2} \\
& +c_{3} k_{2} \tau \sum_{n=1}^{N} \sum_{p=1}^{M_{1}-1} \sum_{q=1}^{M_{2}-1} h_{1} h_{2} \sum_{k=1}^{n} d_{n-k}^{(\alpha)} \nabla_{t} U_{p q}^{k} \nabla_{t} U_{p q}^{n} \\
& +c_{4} \tau \sum_{n=1}^{N} \sum_{p=1}^{M_{1}-1} \sum_{q=1}^{M_{2}-1} h_{1} h_{2} U_{p q}^{n-\frac{1}{2}} \nabla_{t} U_{p q}^{n}
\end{aligned}
$$




$$
\begin{aligned}
= & c_{5} \tau \sum_{n=1}^{N} \sum_{p=1}^{M_{1}-1} \sum_{q=1}^{M_{2}-1} h_{1} h_{2}\left(\delta_{x}^{2}+\delta_{y}^{2}\right) U_{p q}^{n-\frac{1}{2}} \nabla_{t} U_{p q}^{n} \\
& +c_{6} k_{3} \tau \sum_{n=1}^{N} \sum_{p=1}^{M_{1}-1} \sum_{q=1}^{M_{2}-1} h_{1} h_{2} \sum_{k=1}^{n} d_{n-k}^{(\beta)} \nabla_{t}\left(\delta_{x}^{2}+\delta_{y}^{2}\right) U_{p q}^{k} \nabla_{t} U_{p q}^{n} \\
& +\tau \sum_{n=1}^{N} \sum_{p=1}^{M_{1}-1} \sum_{q=1}^{M_{2}-1} h_{1} h_{2} f_{p q}^{n-\frac{1}{2}} \nabla_{t} U_{p q}^{n} .
\end{aligned}
$$

Using Lemma 3, we have

$$
\begin{aligned}
& c_{1} k_{1} \tau \sum_{n=1}^{N} \sum_{p=1}^{M_{1}-1} \sum_{q=1}^{M_{2}-1} h_{1} h_{2}\left[a_{0}^{(\gamma)} \nabla_{t} U_{p q}^{n}-\sum_{k=1}^{n-1}\left(a_{n-k-1}^{(\gamma)}-a_{n-k}^{(\gamma)}\right) \nabla_{t} U_{p q}^{k}-a_{n-1}^{(\gamma)} \phi_{p q}\right] \nabla_{t} U_{p q}^{n} \\
& \geq c_{1} \frac{\tau T^{1-\gamma}}{2 \Gamma(2-\gamma)} \sum_{n=1}^{N} \sum_{p=1}^{M_{1}-1} \sum_{q=1}^{M_{2}-1} h_{1} h_{2}\left(\nabla_{t} U_{p q}^{n}\right)^{2}-c_{1} \frac{a_{1} T^{2-\gamma}}{2 \Gamma(3-\gamma)} \sum_{p=1}^{M_{1}-1} \sum_{q=1}^{M_{2}-1} h_{1} h_{2} \phi_{p q}^{2} \\
& =c_{1} \frac{\tau T^{1-\gamma}}{2 \Gamma(2-\gamma)} \sum_{n=1}^{N}\left\|\nabla_{t} U^{n}\right\|_{0}^{2}-c_{1} \frac{T^{2-\gamma}}{2 \Gamma(3-\gamma)}\|\phi\|_{0}^{2} .
\end{aligned}
$$

For the second term, we obtain

$$
c_{2} \tau \sum_{n=1}^{N} \sum_{p=1}^{M_{1}-1} \sum_{q=1}^{M_{2}-1} h_{1} h_{2}\left(\nabla_{t} U_{p q}^{n}\right)^{2}=c_{2} \tau \sum_{n=1}^{N}\left\|\nabla_{t} U^{n}\right\|_{0}^{2} .
$$

Using Lemma 2, we get

$$
\begin{gathered}
c_{3} k_{2} \tau \sum_{n=1}^{N} \sum_{p=1}^{M_{1}-1} \sum_{q=1}^{M_{2}-1} h_{1} h_{2} \sum_{k=1}^{n} d_{n-k}^{(\alpha)} \nabla_{t} U_{p q}^{k} \nabla_{t} U_{p q}^{n} \\
=c_{3} k_{2} \sum_{n=1}^{N} \sum_{k=1}^{n} d_{n-k}^{\left(\alpha_{m}\right)}\left(\nabla U^{k}, \nabla U^{n}\right) \geq 0 .
\end{gathered}
$$

For the forth term, we have

$$
\begin{gathered}
c_{4} \tau \sum_{n=1}^{N} \sum_{p=1}^{M_{1}-1} \sum_{q=1}^{M_{2}-1} h_{1} h_{2} U_{p q}^{n-\frac{1}{2}} \nabla_{t} U_{p q}^{n}=\frac{c_{4}}{2} \sum_{n=1}^{N}\left(U^{n}+U^{n-1}, U^{n}-U^{n-1}\right) \\
=\frac{c_{4}}{2} \sum_{n=1}^{N}\left(\left\|U^{n}\right\|_{0}^{2}-\left\|U^{n-1}\right\|_{0}^{2}\right)=\frac{c_{4}}{2}\left(\left\|U^{N}\right\|_{0}^{2}-\left\|U^{0}\right\|_{0}^{2}\right) .
\end{gathered}
$$

Applying (3) and using the inequality $a(a-b) \geq \frac{1}{2}\left(a^{2}-b^{2}\right)$, we obtain

$$
\begin{gathered}
c_{5} \tau \sum_{n=1}^{N} \sum_{p=1}^{M_{1}-1} \sum_{q=1}^{M_{2}-1} h_{1} h_{2}\left(\delta_{x}^{2}+\delta_{y}^{2}\right) U_{p q}^{n} \nabla_{t} u_{p q}^{n} \\
=c_{5} \tau \sum_{n=1}^{N}\left(\left(\delta_{x}^{2}+\delta_{y}^{2}\right) U^{n}, \nabla_{t} U^{n}\right)
\end{gathered}
$$




$$
\begin{aligned}
& =-c_{5} \sum_{n=1}^{N}\left\langle\left(\nabla_{x}+\nabla_{y}\right) U^{n},\left(\nabla_{x}+\nabla_{y}\right) U^{n}-\left(\nabla_{x}+\nabla_{y}\right) U^{n-1}\right\rangle \\
& \leq-\frac{c_{5}}{2} \sum_{n=1}^{N}\left(\left|U^{n}\right|_{1}^{2}-\left|U^{n-1}\right|_{1}^{2}\right)=\frac{c_{5}}{2}\left(\left|U^{0}\right|_{1}^{2}-\left|U^{N}\right|_{1}^{2}\right) .
\end{aligned}
$$

Combining (4) and Lemma 2, we have

$$
\begin{aligned}
& c_{6} k_{3} \tau \sum_{n=1}^{N} \sum_{p=1}^{M_{1}-1} \sum_{q=1}^{M_{2}-1} h_{1} h_{2} \sum_{k=1}^{n} c_{n-k}^{(\beta)} \nabla_{t}\left(\delta_{x}^{2}+\delta_{y}^{2}\right) U_{p q}^{k} \nabla_{t} U_{p q}^{n} \\
& =c_{6} k_{3} \tau \sum_{n=1}^{N} \sum_{k=1}^{n} c_{n-k}^{\left(\beta_{)}\right)}\left(\nabla_{t}\left(\left(\delta_{x}^{2}+\delta_{y}^{2}\right) U^{k}\right), \nabla_{t} U^{n}\right) \\
& =-c_{6} k_{3} \tau \sum_{n=1}^{N} \sum_{k=1}^{n} c_{n-k}^{\left(\beta_{)}\right.}\left\langle\nabla_{t}\left(\left(\nabla_{x}+\nabla_{y}\right) U^{k}\right), \nabla_{t}\left(\left(\nabla_{x}+\nabla_{y}\right) U^{n}\right)\right\rangle \leq 0 .
\end{aligned}
$$

For the last term and using the important inequality $a b \leq \varepsilon a^{2}+\frac{b^{2}}{4 \varepsilon}$, we have

$$
\begin{aligned}
\tau \sum_{n=1}^{N} & \sum_{p=1}^{M_{1}-1} \sum_{q=1}^{M_{2}-1} h_{1} h_{2} f_{p q}^{n-\frac{1}{2}} \nabla_{t} U_{p q}^{n} \\
\leq & \tau\left(\frac{c_{1} T^{1-\gamma_{l}}}{2 \Gamma\left(2-\gamma_{l}\right)}+c_{2}\right) \sum_{n=1}^{N} \sum_{p=1}^{M_{1}-1} \sum_{q=1}^{M_{2}-1} h_{1} h_{2}\left(\nabla_{t} U_{p q}^{n-\frac{1}{2}}\right)^{2} \\
& +\frac{\tau}{4\left(\frac{c_{1} T^{1-\gamma_{l}}}{2 \Gamma\left(2-\gamma_{l}\right)}+c_{2}\right)} \sum_{n=1}^{N} \sum_{p=1}^{M_{1}-1} \sum_{q=1}^{M_{2}-1} h_{1} h_{2}\left(f_{p q}^{n-\frac{1}{2}}\right)^{2} \\
= & \tau\left(\frac{c_{1} T^{1-\gamma_{l}}}{2 \Gamma\left(2-\gamma_{l}\right)}+c_{2}\right) \sum_{n=1}^{N}\left\|\nabla_{t} U^{n}\right\|_{0}^{2}+\frac{\tau}{4\left(\frac{c_{1} T^{1-\gamma_{l}}}{2 \Gamma\left(2-\gamma_{l}\right)}+c_{2}\right)} \sum_{n=1}^{N}\left\|f^{n-\frac{1}{2}}\right\|_{0}^{2} .
\end{aligned}
$$

Substituting (13)-(19) into (12), we have

$$
\begin{aligned}
& \tau\left(\frac{c_{1} T^{1-\gamma_{l}}}{2 \Gamma\left(2-\gamma_{l}\right)}+c_{2}\right) \sum_{n=1}^{N}\left\|\nabla_{t} U^{n}\right\|_{0}^{2}-\frac{c_{1} T^{2-\gamma_{l}}}{2 \Gamma\left(3-\gamma_{l}\right)}\|\phi\|_{0}^{2}+\frac{c_{4}}{2}\left(\left\|U^{N}\right\|_{0}^{2}-\left\|u^{0}\right\|_{0}^{2}\right) \\
& \quad \leq \frac{c_{5}}{2}\left(\left|U^{0}\right|_{1}^{2}-\left|U^{N}\right|_{1}^{2}\right) \\
& \quad+\tau\left(\frac{c_{1} T^{1-\gamma_{l}}}{2 \Gamma\left(2-\gamma_{l}\right)}+c_{2}\right) \sum_{n=1}^{N}\left\|\nabla_{t} U^{n}\right\|_{0}^{2}+\frac{\tau}{4\left(\frac{c_{1} T^{1-\gamma_{l}}}{2 \Gamma\left(2-\gamma_{l}\right)}+c_{2}\right)} \sum_{n=1}^{N}\left\|f^{n-\frac{1}{2}}\right\|_{0}^{2},
\end{aligned}
$$

then we have

$$
\begin{aligned}
c_{4}\left\|U^{N}\right\|_{0}^{2}+c_{5}\left|U^{N}\right|_{1}^{2} \\
\leq c_{4}\left\|U^{0}\right\|_{0}^{2}+c_{5}\left|U^{0}\right|_{1}^{2} \\
\quad+\frac{c_{1} T^{2-\gamma_{l}}}{\Gamma\left(3-\gamma_{l}\right)}\|\phi\|_{0}^{2}+\frac{T}{\left(\frac{c_{1} T^{1-\gamma_{l}}}{2 \Gamma\left(2-\gamma_{l}\right)}+2 c_{2}\right)} \max _{1 \leq n \leq N}\left\|f^{n-\frac{1}{2}}\right\|_{0}^{2} .
\end{aligned}
$$


Then we have

$$
\begin{aligned}
\left\|U^{N}\right\|_{1}^{2} \leq & \left\|U^{0}\right\|_{1}^{2} \\
& +c_{1} \frac{T^{2-\gamma_{l}}}{\Gamma\left(3-\gamma_{l}\right)}\|\phi\|_{0}^{2}+\frac{T}{\left(\frac{c_{1} T^{1-\gamma_{l}}}{\Gamma\left(2-\gamma_{l}\right)}+2 c_{2}\right)} \max _{1 \leq n \leq N}\left\|f^{n-\frac{1}{2}}\right\|_{0}^{2},
\end{aligned}
$$

which means that scheme (11) is unconditionally stable.

\subsection{Convergence}

Theorem 3 Define $u^{n}$ and $U^{n}$ as the exact solution and numerical solution vectors of scheme (11), respectively. Suppose that the solution $u(x, y, t) \in C_{x, y, t}^{4,4,3}(\Omega)$, then there exists a positive constant $C$ independent of $h$ and $\tau$ such that

$$
\left\|u^{n}-U^{n}\right\|_{1} \leq C \sqrt{\frac{T L_{p} L_{q}}{\frac{2 c_{1} T^{1-\gamma}}{\Gamma(2-\gamma)}+4 b_{1}}}\left(\tau^{\min (3-\gamma, 2-\alpha, 2-\beta)}+h_{1}^{2}+h_{2}^{2}\right) .
$$

Proof Similar to Theorem 2, it is easy to prove.

\section{Numerical example}

In this section, we carry out numerical experiments using the proposed finite difference schemes to illustrate our theoretical statements. Consider the following multi-term time fractional diffusion equation:

$$
\left\{\begin{array}{l}
c_{1} D_{t}^{\gamma} u(x, y, t)+c_{2} \frac{\partial u(x, y, t)}{\partial t}+c_{3} D_{t}^{\alpha} u(x, y, t)+c_{4} u(x, y, t) \\
\quad=c_{5} \Delta u(x, y, t)+c_{6} D_{t}^{\beta} \Delta u(x, y, t)+f(x, y, t), \\
u(x, y, 0)=\sin (\pi x) \sin (\pi y), \quad u_{t}(x, y, 0)=0, \quad 0 \leq x \leq a, 0 \leq y \leq b, \\
u(0, y, t)=0, \quad u(a, y, t)=0 ; \quad u(x, 0, t)=0, \quad u(x, b, t)=0, \quad 0 \leq t \leq T,
\end{array}\right.
$$

where $(x, y, t) \in[0, a] \times[0, b] \times[0, T], 0<\alpha, \beta<1,1<\gamma<2$, the source term is $f(x, y, t)=$ $\sin (\pi x) \sin (\pi y)\left[\frac{\Gamma(p+1) t^{p-\gamma}}{\Gamma(p+1-\gamma)}+p t^{p}+\frac{\Gamma(p+1) t^{p-\alpha}}{\Gamma(p+1-\alpha)}+\left(1+2 \pi^{2}\right)\left(t^{p}+1\right)+\frac{2 \pi^{2} \Gamma(p+1) t^{p-\delta}}{\Gamma(p+1-\delta)}\right]$. And the exact solution is $u(x, y, t)=\left(t^{p}+1\right) \sin (\pi x) \sin (\pi y), p \geq 2$.

Case 1: In this simulation, we choose $c_{1}=c_{2}=c_{3}=c_{4}=c_{5}=c_{6}=1, a=b=1, h_{1}=h_{2}=h$. Firstly, we use the implicit finite difference scheme (11) to solve the equation, and the numerical results are given in Tables 1 and 2. Table 1 shows the $L_{2}$ error and $L_{\infty}$ error and the convergence order of $h$ for different $\gamma, \alpha, \beta$ with $\tau=1 / 1000$ at $t=1$. Table 2 shows the $L_{2}$ error and $L_{\infty}$ error and the convergence order of $\tau$ for different $\gamma, \alpha, \beta$ with $\tau^{\min \{3-\gamma, 2-\alpha, 2-\beta\}} \approx h^{2}$ at $t=1$.

From the tables, we can find that the numerical results are in good agreement with the exact solution and reach the accuracy of $\tau^{\min \{3-\gamma, 2-\alpha, 2-\beta\}}+h^{2}$ order, which demonstrates the effectiveness of our numerical method and confirms the theoretical analysis.

Case 2: In this calculation, we choose $a=b=1, h_{1}=h_{2}=h=1 / 20, \tau=1 / 100$. In order to observe the effects of different physical parameters on the velocity field, we plot some fig- 
Table 1 The spacial error and convergence order of the scheme for different $\gamma, \alpha$, $\beta$ with $\tau=1 / 1000$

\begin{tabular}{lllll}
\hline$\gamma=1.7, \alpha=0.8, \beta=0.6$ & $L_{2}$ error & Order & $L_{\infty}$ error & Order \\
\hline$h=1 / 4$ & $3.5954 \mathrm{E}-02$ & & $7.1908 \mathrm{E}-02$ & \\
$h=1 / 8$ & $8.8281 \mathrm{E}-03$ & 2.03 & $1.7656 \mathrm{E}-02$ & 2.03 \\
$h=1 / 16$ & $2.1957 \mathrm{E}-03$ & 2.01 & $4.3914 \mathrm{E}-03$ & 2.01 \\
$h=1 / 32$ & $5.4676 \mathrm{E}-04$ & 2.01 & $1.0935 \mathrm{E}-03$ & 2.01 \\
$h=1 / 64$ & $1.3510 \mathrm{E}-04$ & 2.02 & $2.7020 \mathrm{E}-04$ & 2.02 \\
\hline \hline $\boldsymbol{\gamma}=1.5, \alpha=0.6, \beta=0.4$ & $L_{2}$ error & Order & $L_{\infty}$ error & Order \\
\hline$h=1 / 4$ & $3.5339 \mathrm{E}-02$ & & $7.0678 \mathrm{E}-02$ & \\
$h=1 / 8$ & $8.6774 \mathrm{E}-03$ & 2.03 & $1.7355 \mathrm{E}-02$ & 2.03 \\
$h=1 / 16$ & $2.1590 \mathrm{E}-03$ & 2.01 & $4.3181 \mathrm{E}-03$ & 2.01 \\
$h=1 / 32$ & $5.3849 \mathrm{E}-04$ & 2.00 & $1.0770 \mathrm{E}-03$ & 2.00 \\
$h=1 / 64$ & $1.3392 \mathrm{E}-04$ & 2.01 & $2.6784 \mathrm{E}-04$ & 2.01 \\
\hline \hline $\boldsymbol{\gamma}=1.2, \alpha=0.4, \beta=0.2$ & $L_{2}$ error & Order & $L_{\infty}$ error & Order \\
\hline$h=1 / 4$ & $3.4841 \mathrm{E}-02$ & & $6.9682 \mathrm{E}-02$ & \\
$h=1 / 8$ & $7.7561 \mathrm{E}-03$ & 2.03 & $1.7108 \mathrm{E}-02$ & 2.03 \\
$h=1 / 16$ & $1.9248 \mathrm{E}-03$ & 2.01 & $4.2575 \mathrm{E}-03$ & 2.01 \\
$h=1 / 32$ & $4.7517 \mathrm{E}-04$ & 2.02 & $1.0628 \mathrm{E}-03$ & 2.00 \\
$h=1 / 64$ & $1.1326 \mathrm{E}-04$ & 2.00 & $2.6526 \mathrm{E}-04$ & 2.00 \\
\hline
\end{tabular}

Table 2 The temporal error and convergence order of the scheme for different $\gamma, \alpha, \beta$ with $\tau^{\min \{3-\gamma, 2-\alpha, 2-\beta\}} \approx h^{2}$

\begin{tabular}{lllll}
\hline$\gamma=1.7, \alpha=0.8, \beta=0.6$ & $L_{2}$ error & Order & $L_{\infty}$ error & Order \\
\hline$\tau=1 / 20$ & $1.5314 \mathrm{E}-02$ & & $3.0629 \mathrm{E}-02$ & \\
$\tau=1 / 40$ & $6.7733 \mathrm{E}-03$ & 1.18 & $1.3138 \mathrm{E}-02$ & 1.22 \\
$\tau=1 / 80$ & $2.7946 \mathrm{E}-03$ & 1.28 & $5.5893 \mathrm{E}-03$ & 1.23 \\
$\tau=1 / 160$ & $1.2456 \mathrm{E}-03$ & 1.17 & $2.4773 \mathrm{E}-03$ & 1.17 \\
$\tau=1 / 320$ & $5.3792 \mathrm{E}-04$ & 1.21 & $1.0758 \mathrm{E}-03$ & 1.20 \\
\hline \hline$\gamma=1.5, \alpha=0.6, \beta=0.4$ & $L_{2}$ error & Order & $L_{\infty}$ error & Order \\
\hline$\tau=1 / 20$ & $8.3425 \mathrm{E}-03$ & & $1.6685 \mathrm{E}-02$ & \\
$\tau=1 / 40$ & $3.1492 \mathrm{E}-03$ & 1.41 & $6.2069 \mathrm{E}-03$ & 1.43 \\
$\tau=1 / 80$ & $1.2085 \mathrm{E}-03$ & 1.38 & $2.4035 \mathrm{E}-03$ & 1.37 \\
$\tau=1 / 160$ & $4.6230 \mathrm{E}-04$ & 1.39 & $9.2459 \mathrm{E}-04$ & 1.38 \\
$\tau=1 / 320$ & $1.7080 \mathrm{E}-04$ & 1.44 & $3.4159 \mathrm{E}-04$ & 1.44 \\
\hline \hline $\boldsymbol{\gamma}=1.2, \alpha=0.4, \beta=0.2$ & $L_{2}$ error & Order & $L_{\infty}$ error & Order \\
\hline$\tau=1 / 20$ & $4.3678 \mathrm{E}-03$ & & $8.5587 \mathrm{E}-03$ & \\
$\tau=1 / 40$ & $1.4595 \mathrm{E}-03$ & 1.58 & $2.8991 \mathrm{E}-03$ & 1.56 \\
$\tau=1 / 80$ & $4.8352 \mathrm{E}-04$ & 1.59 & $9.6484 \mathrm{E}-04$ & 1.59 \\
$\tau=1 / 160$ & $1.5651 \mathrm{E}-04$ & 1.63 & $3.1303 \mathrm{E}-04$ & 1.62 \\
$\tau=1 / 320$ & $5.2751 \mathrm{E}-05$ & 1.57 & $1.0550 \mathrm{E}-04$ & 1.57 \\
\hline
\end{tabular}

ures to demonstrate the dynamic characteristics of the generalized non-Newtonian fluid. Figure 1 shows the influence of time on the velocity and the flow velocity increase with $t=0.5$ and $t=1$ respectively, and coefficients $c_{i}=1, i=1, \ldots, 6$. In order to show the difference clearly, we choose $y=1$, and the variations of $u(x, y, t)$ with $x$ for different values of $\lambda, \alpha, \beta, p, c_{i}=1, i=1, \ldots, 6$ at a fixed time $(t=1)$ are illustrated in Figs. 2-5. From the figures, we can conclude that the coefficients $c_{i}=1, i=1, \ldots, 6$, parameter $p$, and the fractional order $\gamma, \alpha, \beta$ have effects on the velocity function $u(x, y, t)$.

\section{Conclusion}

In this paper, we proposed the finite difference method, based on the Crank-Nicolson method, to solve the multi-term time fractional generalized non-Newtonian fluid equa- 


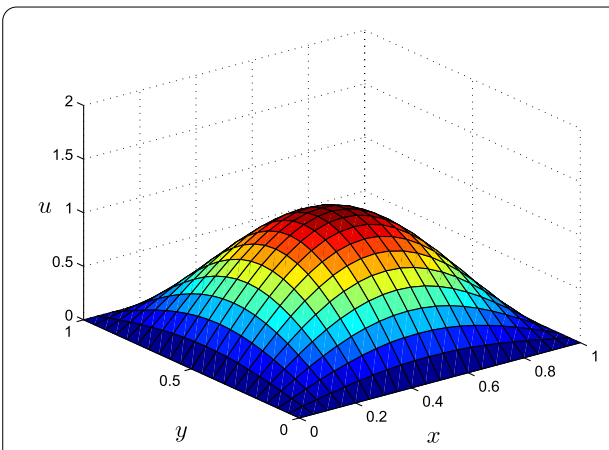

(a) $t=0.5$

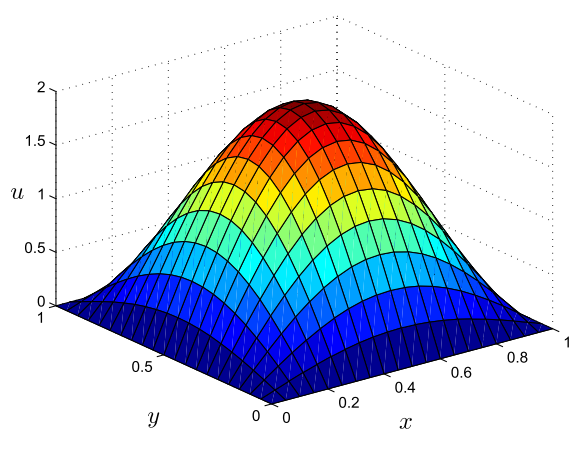

(b) $t=1$

Figure 1 Numerical solution profiles of velocity $u(x, y, t)$ with $\gamma=1.7, \alpha=0.8, \beta=0.6$

Figure 2 Numerical solution profiles of velocity $u(x$, $y, t)$ with different $\gamma, \alpha, \beta$ with $c_{1}=1, c_{2}=5, c_{3}=3$, $c_{4}=1, c_{5}=5, c_{6}=5, p=3$
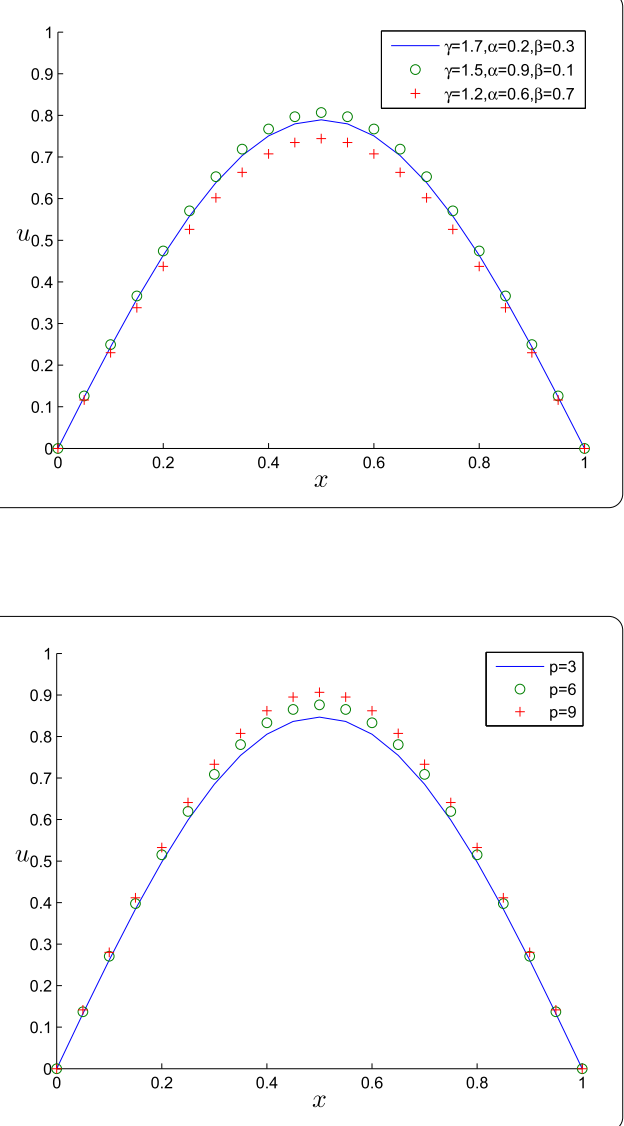

Figure 3 Numerical solution profiles of velocity $u(x$, $y, t)$ with different $p$ with $\gamma=1.6, \alpha=0.5, \beta=0.3$, $c_{1}=1, c_{2}=5, c_{3}=3, c_{4}=2, c_{5}=4, c_{6}=3$

tion. This is a finite difference scheme with accuracy of $O\left(\tau^{\min \{3-\gamma, 2-\alpha, 2-\beta\}}+h_{x}^{2}+h_{y}^{2}\right)$. And we established the unconditional stability and convergence analysis for this implicit difference scheme. Numerical experiments were exhibited to verify the effectiveness and reliability of this method. We can conclude that this numerical method is robust and can be extended to other multi-term time fractional diffusion equations, multi-term time fractional diffusion-wave equations. 
Figure 4 Numerical solution profiles of velocity $u(x$, $y, t)$ with different $c_{1}, c_{3}, c_{6}$ with $\gamma=1.2, \alpha=0.5$, $\beta=0.8, c_{2}=c_{4}=c_{5}=1, p=3$

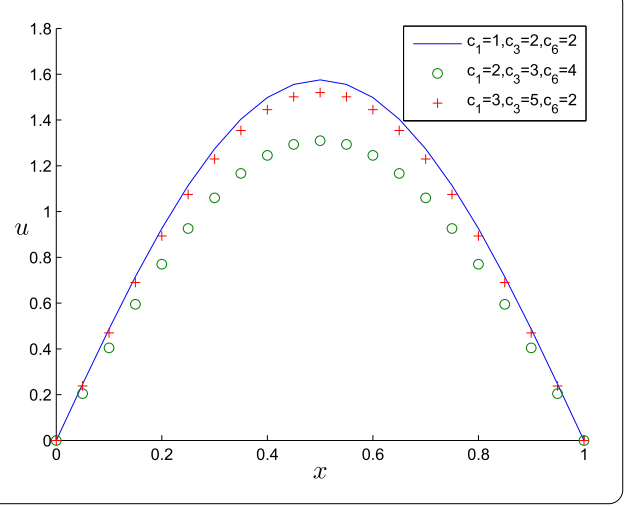

Figure 5 Numerical solution profiles of velocity $u(x$, $y, t)$ with different $c_{2}, c_{4}, c_{5}$ with $\gamma=1.2, \alpha=0.5$, $\beta=0.8, c_{1}=c_{3}=c_{6}=1, p=3$

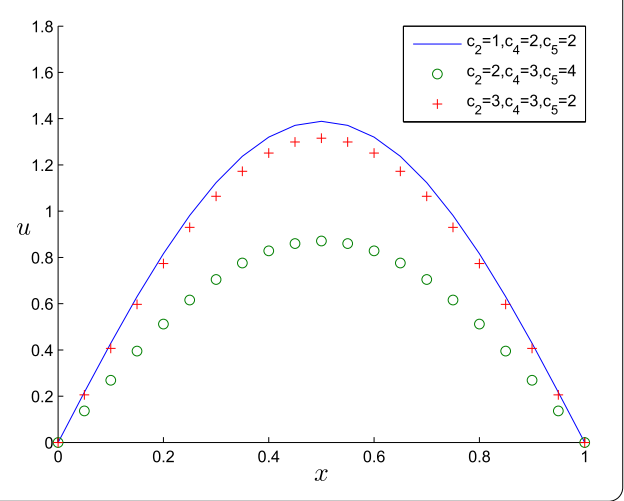

\section{Funding}

This article is supported by the National Natural Science Foundation of China (Nos. 11501082, 11801060), the Natural Science Foundation of Shandong Province (No. ZR2016AQ07), and the Postdoctoral Science Foundation of Jiangsu Province (Grant No. 1402046C).

\section{Competing interests}

The authors declare that they have no competing interests.

\section{Authors' contributions}

All authors contributed equally to each part of this work. All authors read and approved the final manuscript.

\section{Author details}

${ }^{1}$ School of Mathematical Sciences, Dezhou University, Dezhou, China. ${ }^{2}$ Institute of Soft Matter Mechanics, Department of Engineering Mechanics, Hohai University, Jiangsu, China. ${ }^{3}$ School of Mathematical Sciences, Queensland University of Technology, Brisbane, Australia.

\section{Publisher's Note}

Springer Nature remains neutral with regard to jurisdictional claims in published maps and institutional affiliations.

\section{Received: 17 July 2018 Accepted: 8 November 2018 Published online: 30 November 2018}

\section{References}

1. Meltzler, R., Klafter, J.: The random walk's guide to anomalous diffusion: a fractional dynamics approach. Phys. Rep. $339,1-77(2000)$

2. Margulis, L.: Fractional Calculus and Waves in Linear Viscoelasticity and Introduction to Mathematical Models. World Scientific, Singapore (2010)

3. Atangana, A., Hammouch, Z., Mophou, G., Owolabi, K.M.: Focus point on modelling complex real-world problems with fractal and new trends of fractional differentiation. Eur. Phys. J. Plus 133, 315 (2018)

4. Scalas, E., Gorenflo, R., Mainardi, F.: Fractional calculus and continuous-time finance. Phys. A, Stat. Mech. Appl. 284 376-384 (2000)

5. Leonenko, N., Meerschaert, M., Sikorskii, A.: Fractional Pearson diffusions. J. Math. Anal. Appl. 403, 532-546 (2013)

6. Liu, F., Zhuang, P., Liu, Q.: Numerical Methods of Fractional Partial Differential Equations and Applications. Science Press, Bejing (2015) 
7. Yang, Q.Q., Turner, I., Liu, F.W., llić, M.: Novel numerical methods for solving the time-space fractional diffusion in two-dimensions. SIAM J. Sci. Comput. 33, 1159-1180 (2011)

8. Jajarmi, A., Hajipour, M., Mohammadzadeh, E., Baleanu, D.: A new approach for the nonlinear fractional optimal control problems with external persistent disturbances. J. Franklin Inst. 389, 1117-1127 (2012)

9. Jajarmi, A., Baleanu, D.: Suboptimal control of fractional-order dynamic systems with delay argument. J. Vib. Control 24, 2430-2446 (2018)

10. Baleanu, D., Jajarmi, A., Bonyah, E., Hajipour, M.: New aspects of the poor nutrition in the life cycle within the fractional calculus. Adv. Differ. Equ. 2018, 230 (2018)

11. Jajarmi, A., Baleanu, D.: A new fractional analysis on the interaction of HIV with CD4 ${ }^{+}$T-cells. Chaos Solitons Fractals $113,221-229(2018)$

12. Jiang, H., Liu, F.W., Turner, I., Burrage, K.: Analytical solutions for the multi-term time-fractional diffusion-wave/diffusion equations in a finite domain. Comput. Math. Appl. 64, 3377-3388 (2012)

13. Daftardar-Gejji, V., Bhalekar, S.: Boundary value problems for multi-term fractional differential equations. J. Math. Anal. Appl. 345, 754-765 (2008)

14. Jin, B.T., Lazarov, R., Liu, Y., Zhou, Z:: The Galerkin finite element method for a multi-term time-fractional diffusion equation. J. Comput. Phys. 281, 825-843 (2015)

15. Liu, F.W., Meerschaert, M.M., McGough, R.J., Zhuang, P.H., Liu, Q.X.: Numerical methods for solving the multi-term time fractional wave-diffusion equation. Fract. Calc. Appl. Anal. 16, 9-25 (2013)

16. Salehi, R.: A meshless point colocation method for 2-D multi-term time fractional diffusion-wave equation. Numer. Algorithms 74, 1145-1168 (2017)

17. Hao, Z.P., Lin, G.: Finite difference schemes for multi-term time-fractional mixed diffusion-wave equations. Preprint

18. Metzler, R., Kalfter, J., Sokolov, I.M.: Anomalous transport in external fields: continuous time random walks and fractional diffusion equations extended. Phys. Rev. E 58, 1621 (1998)

19. Schumer, R., Benson, D.A., Meerschaert, M.M., Baeumer, B.: Fractal mobile/immobile solute transport. Water Resour. Res. 39, 1296 (2003)

20. Kelly, J.F., McGough, R.J., Meerschaert, M.M.: Analytical time-domain Green's functions for power-law media. J. Acoust. Soc. Am. 124, 2861-2872 (2008)

21. Bagley, R.L., Torvik, P.J.: A theorectical basis for the application of fractional calculus to viscoelasticity. J. Rheol. 27, 201-210 (1983)

22. Makris, N., Constantinou, M.C.: Fractional-derivative Maxwell model for viscous danmpers. J. Struct. Eng. 117 2708-2724 (1991)

23. Qi, H.T. Xu, M.Y.: Some unsteady unidirectional flows of a generalized Oldroyd-B fluid with fractional derivative. Appl. Math. Model. 33, 4184-4191 (2009)

24. Jiang, Y.T., Qi, H.T., Xu, H.Y., Jiang, X.Y.: Transient electroosmotic slip flow of fractional Oldroyd-B fluids. Microfluid. Nanofluid. 21, 7 (2017)

25. Sutton, G.W., Sherman, A.: Engineering Magnetohydrodynamics. McGraw-Hill, New York (1965)

26. Khan, M., Maqbool, K., Hayat, T.: Influence of hall current on the flows of a generalzied Oldroyd-B fluid in a porous space. Acta Mech. 184, 1-13 (2006)

27. Zheng, L.C., Liu, Y.Q., Zhang, X.X.: Slip effects on MHD flow of a generalized Oldroyd-B fluid with fractional derivative. Nonlinear Anal., Real World Appl. 13, 513-523 (2012)

28. Jiang, X.Y., Qi, H.T.: Thermal wave model of bioheat transfer with modified Riemann-Liouville fractional derivative. J. Phys. A, Math. Theor. 45, 831-842 (2012)

29. Fetecau, C., Fetecau, C., Kamran, M., Vieru, D.: Exact solutions for the flow of a generalized Oldroyd-B fluid induced by a constantly accelerating plate between side walls perpendicular to the plate. J. Non-Newton. Fluid Mech. 156, 189-201 (2009)

30. Zaky, M., Doha, E.H., Taha, T.M., Baleanu, D.: New recursive approximations for variable-order fractional operators with applications. Math. Model. Anal. 23, 227-239 (2018)

31. Bhrawy, A.H., Doha, E.H., Baleanu, D., Hafez, R.M.: A highly accurate Jacobi collocation algorithm for systems of high-order linear differential-difference equations with mixed initial conditions. Math. Methods Appl. Sci. 38, 3022-3032 (2015)

32. Ding, H.F., Li, C.P.: Fractional-compact numerical algorithms for Riesz spatial fractional reaction-dipersion equations. Fract. Calc. Appl. Anal. 20, 722-764 (2017)

33. Alikhanov, A.A.: A new difference scheme for the time fractional diffusion equation. J. Comput. Phys. 280, 424-438 (2015)

34. Gao, G.H., Sun, Z.Z., Zhang, H.W.: A new fractional numerical differentiation formula to approximate the Caputo fractional derivative and its applications. J. Comput. Phys. 259, 33-50 (2014)

35. Liu, Y., Du, Y.W., Li, H., Li, J.C., He, S.G.: A two-grid mixed finite element method for a nonlinear fourth order reaction diffusion problem with time-fractional derivative. Comput. Math. Appl. 70, 2474-2492 (2015)

36. Zhao, Y.M., Bu, W.P., Huang, J.F., Liu, D.Y., Tang, Y.F.: Finite element method for two-dimensional space-fractonal advection dispersion equations. Appl. Math. Comput. 257, 553-565 (2015)

37. Simmons, A., Yang, Q.Q., Moroney, T.: A finite volume method for two-sided fractional diffusion equations on non-uniform meshes. J. Comput. Phys. 335, 747-759 (2017)

38. Jia, J.H., Wang, H.: A fast finite volume method for conservative space-fractional diffusion equations in convex domains. J. Comput. Phys. 310, 63-84 (2016)

39. Bhrawy, A.H., Abdelkawy, M.A. Baleanu, D. Amin, A.Z.M. A spectral technique for solving two-dimensional fractional integral equations with weakly singular kernel. Hacet. J. Math. Stat. 47, 553-566 (2018)

40. Zayernouri, M., Karniadakis, G.E.: Fractional spectral collocation method. SIAM J. Sci. Comput. 36, 40-62 (2014)

41. Zeng, F., Liu, F., Li, C.P., Burrage, K., Turner, I., Anh, V.: A Crank-Nicolson adi spectral method for a two-dimensional Riesz space fractional nonlinear reaction-diffusion equation. SIAM J. Numer. Anal. 52, 2599-2622 (2014)

42. Bazhlekova, E., Bazhlekov, l.: Viscoelastic flows with fractional derivative models: computational approach by convolutional calculus of Dimovski. Fract. Calc. Appl. Anal. 17, 954-976 (2014)

43. Feng, L.B., Liu, F.W., Turner, I., Zhuang, P.H.: Numerical methods and analysis for simulating the flow of a generalized Oldroyd-B fluid between two infinite parallel rigid plates. Int. J. Heat Mass Transf. 115, 1309-1320 (2017) 
44. Karatay, I., Kale, N., Bayramoglu Erguner, S.R.: A new difference scheme for time fractional heat equations based on the Crank-Nicholson method. Fract. Calc. Appl. Anal. 16, 892-910 (2013)

45. Hilfer, R.: Applications of Fractional Calculus in Physics. World Scientific, Singapore (2000)

46. Podlubny, I.: Fractional Differential Equations. Academic Press, San Diego (1999)

47. Liu, J.C., Li, H., Liu, Y.: A new fully discrete finite difference/element approxiamation for fractional cable equation. J. Appl. Math. Comput. 52, 345-361 (2016)

48. Feng, L., Liu, F., Turner, I., Zheng, L.: Novel numerical analysis of multi-term time fractional viscoelastic non-Newtonian fluid models for simulating unsteady MHD Couette flow of a generalized Oldroyd-B. Fract. Calc. Appl. Anal. 21(4), 1073-1103 (2018)

49. Böttcher, A., Silbermann, B.: Analysis of Toeplitz Operators, 2nd edn. Springer, Berlin (2005)

50. Sun, Z.Z., Wu, X.N.: A fully discrete difference scheme for a diffusion-wave system. Appl. Numer. Math. 56, 193-209 (2006)

Submit your manuscript to a SpringerOpen ${ }^{\circ}$ journal and benefit from:

- Convenient online submission

Rigorous peer review

- Open access: articles freely available online

- High visibility within the field

- Retaining the copyright to your article

Submit your next manuscript at $\boldsymbol{\triangleright}$ springeropen.com 